\title{
Parameters of two low-mass contact eclipsing binaries near the short-period limit
}

\author{
M. E. Lohr ${ }^{1}$, S. T. Hodgkin ${ }^{2}$, A. J. Norton ${ }^{1}$, and U. C. Kolb ${ }^{1}$ \\ ${ }^{1}$ Department of Physical Sciences, The Open University, Walton Hall, Milton Keynes MK7 6AA, UK \\ e-mail: Marcus.Lohr@open.ac.uk \\ 2 Institute of Astronomy, Madingley Road, Cambridge CB3 OHA, UK \\ Received 21 May 2013 / Accepted 31 January 2013
}

ABSTRACT

\begin{abstract}
The two objects 1SWASP J150822.80-054236.9 and 1SWASP J160156.04+202821.6 were initially detected from their SuperWASP archived light curves as candidate eclipsing binaries with periods close to the short-period cut-off of the orbital period distribution of main-sequence binaries, at $\sim 0.2 \mathrm{~d}$. Using spectroscopic data from the Isaac Newton Telescope in La Palma, Canary Islands, we here confirm them as double-lined spectroscopic and eclipsing binaries, in contact configuration. Following modelling of their visual light curves and radial velocity curves, we determine their component and system parameters to precisions between $\sim 2$ and $11 \%$. The first system contains components of 1.07 and $0.55 M_{\odot}$, with radii of 0.90 and $0.68 R_{\odot}$, respectively; its primary exhibits pulsations with a period of $1 / 6$ of the orbital period of the system. The latter contains components of 0.86 and $0.57 M_{\odot}$, with radii of 0.75 and $0.63 R_{\odot}$, respectively.
\end{abstract}

Key words. binaries: close - binaries: eclipsing - binaries: spectroscopic - stars: individual: 1SWASP J150822.80-054236.9 stars: individual: 1SWASP J160156.04+202821.6

\section{Introduction}

The orbital period distribution of main-sequence binary stars exhibits a fairly sharp lower limit at around $0.2 \mathrm{~d}$ (Rucinski 1992, 2007; Szymański et al. 2001; Paczyński et al. 2006), the cause of which is the subject of ongoing research, see for example Stepień (2006), Stepień \& Gazeas (2012), Jiang et al. (2012). However, despite the inherent interest in this region of parameter space, relatively few eclipsing binaries (EB) have been discovered with periods that are near the cut-off point. This motivated a search of the archive of the SuperWASP project (Wide Angle Search for Planets: Pollacco et al. 2006) for EB candidates with apparent periods $<20000 \mathrm{~s}(\sim 0.2315 \mathrm{~d})$, reported in Norton et al. (2011). Fifty-three candidates were found, 48 of which were new discoveries at the time. A subsequent search of these candidate EBs for evidence of period change (Lohr et al. 2012) corrected the periods of nine EBs to values slightly higher than $20000 \mathrm{~s}$, but still with $<22600 \mathrm{~s}(\sim 0.2616 \mathrm{~d})$. A more rigorous search of the SuperWASP archive (Lohr et al. 2013) then detected 143 candidate EBs with periods $<20000$ s, including 97 new discoveries since the publication of Norton et al. (2011), and measured significant period changes in 74 candidates.

Here, spectroscopic data allow us to confirm two of these candidates as double-lined EBs in contact configuration (W UMa-type variables): 1SWASP J150822.80-054236.9 (J150822) and 1SWASP J160156.04+202821.6(J160156). Both were initially identified in Norton et al. (2011); the period of J150822 was revised upwards to $22469.2 \mathrm{~s}$ in Lohr et al. (2012) and therefore it did not appear in Lohr et al. (2013). We report system and component parameters obtained for these EBs by simultaneous modelling of their SuperWASP light curves and radial velocities. These are of interest for the study of low-mass dwarfs and W UMa systems in general, and of very short period binaries specifically.

\section{Observations}

\subsection{Photometry}

The SuperWASP archive contains 30131 photometric points for J150822, taken between 5 March 2008 and 29 March 2011. For J160156 there are 14651 observations, made between 2 May 2004 and 21 February 2011. Sys-Rem-corrected fluxes (Tamuz et al. 2005; Mazeh et al. 2006) from the 3.5 pixelradius photometric aperture (the middle of three available apertures) were used to construct the light curves used here, which correspond approximately to the Johnson $V$ band. Periods and period-change measurements were obtained using a customwritten IDL program, as described in Lohr et al. (2013), and the binned averaged phase-folded data produced high-precision phased light curves (Figs. 1 to 4). A small secular period decrease $\left(-0.055 \mathrm{~s} \mathrm{y}^{-1}\right)$ was measured for J150822, and a slightly larger secular period increase $\left(0.094 \mathrm{~s} \mathrm{y}^{-1}\right)$ for J160156; both values are fairly unexceptional for variables of this type (Lohr et al. 2013). The scatter here is comparable to that seen in other SuperWASP light curves for objects of similar magnitude, and it is unlikely that either period variation over time or flux variability caused by surface spots significantly contribute to it. The uncertainties on the means in the light curves used in subsequent modelling were given by the standard deviation of points in each bin, divided by the square root of the number of observations per bin, i.e. $\sigma / \sqrt{n}$.

\subsection{Spectroscopy}

Thirty-six long-slit spectra were obtained for J150822 and 28 for J160156 with the Intermediate Dispersion Spectrograph (IDS) on the $2.5 \mathrm{~m}$ Isaac Newton Telescope (INT) at La Palma in the Canary Islands. The observations for the two stars were interspersed with each other and cover three consecutive nights 


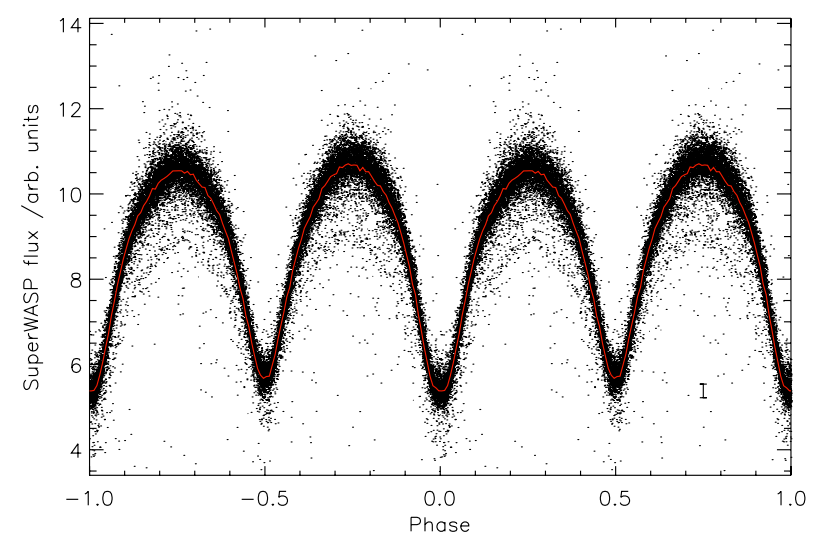

Fig. 1. SuperWASP light curve for J150822, folded at a period of 22469.219 s, with the binned mean curve overplotted. A typical uncertainty range for a single observation is shown. These fluxes correspond to a visual magnitude range of $\sim 12.4-13.2$.

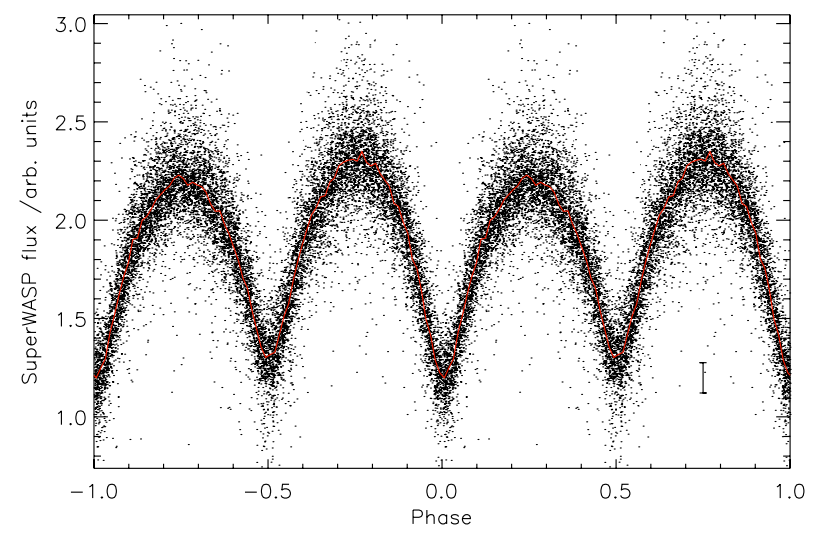

Fig. 2. SuperWASP light curve for J160156, folded at a period of $19572.136 \mathrm{~s}$, with the binned mean curve overplotted. A typical uncertainty range for a single observation is shown. These fluxes correspond to a visual magnitude range of $\sim 14.1-14.8$.

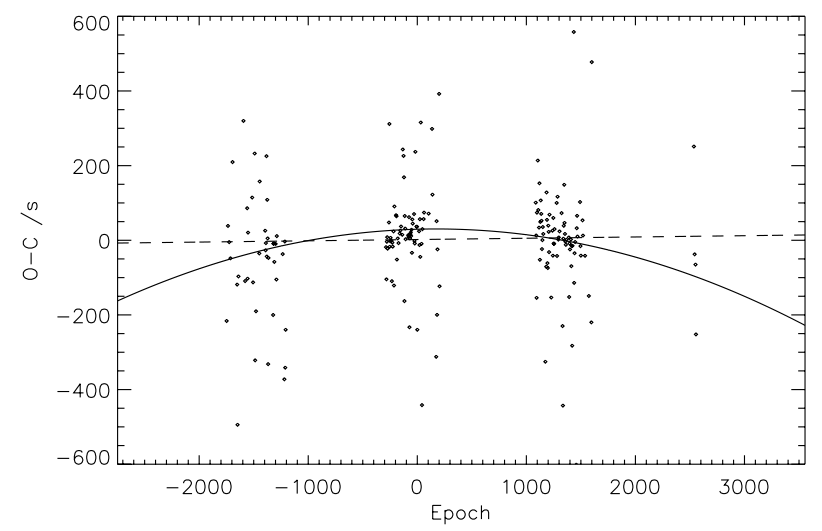

Fig. 3. Observed minus calculated (O-C) diagram for J150822, with best linear (dashed line; $\chi^{2}=5.25$ ) and quadratic (solid curve; $\chi^{2}=$ 4.87) fits overplotted. For clarity of presentation, uncertainties are not shown. Period decrease of $-0.055 \pm 0.006 \mathrm{~s} \mathrm{y}^{-1}$ is indicated $(\sigma=8)$.

(11-13 March 2012) to optimise phase coverage. Exposures were 300 or $600 \mathrm{~s}$ to allow for the short orbital periods involved, and a wavelength range of $\sim 7915-9040 \AA$ was chosen, which covers the Ca II triplet. The RED+2 CCD and R1200R gratings were used, providing a resolution of $0.51 \AA$ per pixel. $\mathrm{S} / \mathrm{N}$ values of $\sim 40-50$ were obtained around quadrature for J150822,

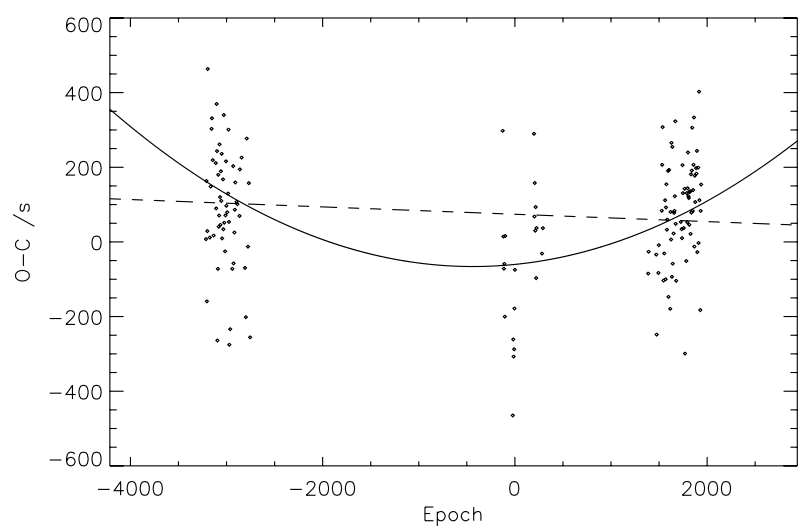

Fig. 4. O-C diagram for J160156, with best linear (dashed line; $\chi^{2}=$ 1.87 ) and quadratic (solid curve; $\chi^{2}=1.64$ ) fits overplotted. For clarity of presentation, uncertainties are not shown. Period increase of $+0.094 \pm 0.015 \mathrm{~s} \mathrm{y}^{-1}$ is indicated $(\sigma=6)$.

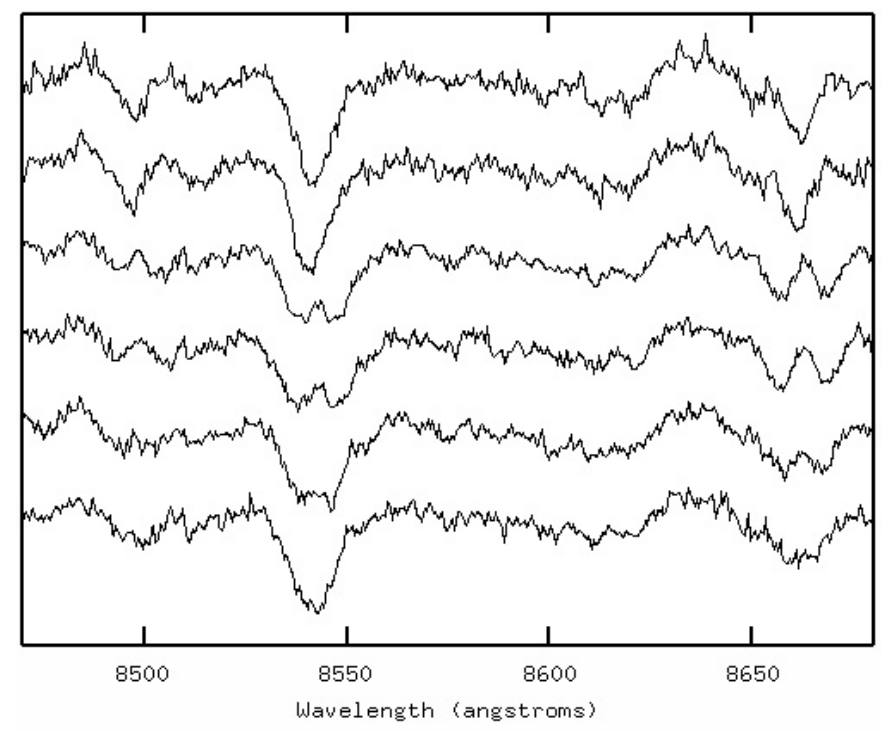

Fig. 5. Selected spectra in the region of Ca II triplet (laboratory wavelengths: $8498.03,8542.09$ and $8662.14 \AA$ ) for J150822, taken from the final night of observations. Line splitting is readily apparent for all three calcium lines.

and $~ 30-40$ for J160156. The spectra were flat-fielded, biascorrected and optimally extracted using standard IRAF routines, and were calibrated using $\mathrm{CuArNe}$ arc lamp exposures.

Line splitting was clearly observable by eye (Figs. 5 and 6) and was used to estimate the phase. A suitable synthetic comparison spectrum was then selected by cross-correlation with a phase 0 program spectrum; the best-matching template for both objects had a temperature of $4500 \mathrm{~K}$. Radial velocities (RVs) were measured by cross-correlation with the template using the IRAF task FXCOR. Uncertainties were minimized by excluding the broadest $\mathrm{Ca}$ II line from consideration. Improved phase determinations were obtained by fitting sinusoidal functions to the $\mathrm{RV}$ curves to locate cross-over points corresponding to phases 0 and 0.5 .

These phasings were then compared with the predictions of the SuperWASP linear and quadratic ephemerides (implying constant periods and secular period change, respectively), and with internal simultaneous low-resolution light curves extracted from the spectra themselves (by evaluating a continuum fit at $8500 \AA$ ), and were found to be substantially self-consistent 


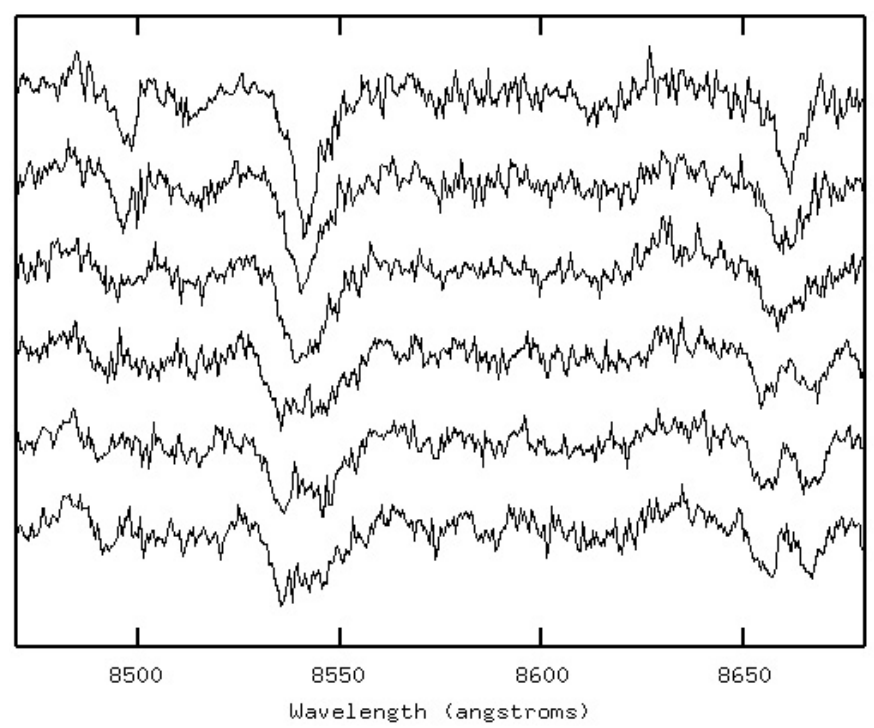

Fig. 6. Selected spectra in region of Ca II triplet for J160156, taken from final night of observations. Line splitting is most obvious for the two calcium lines at longer wavelengths.

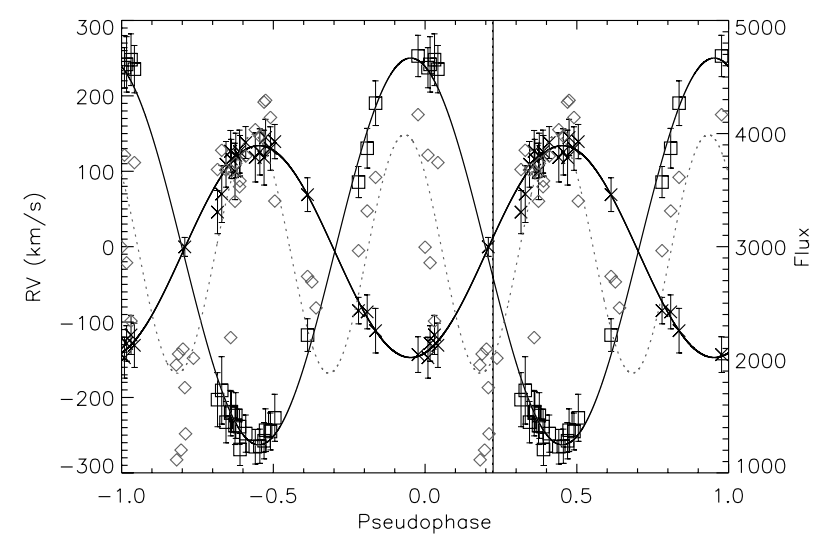

Fig. 7. Radial velocities for J150822 (crosses indicate primary component, squares secondary) with preliminary fits (solid curves) used to obtain correct phasing of observations. Also shown are a light curve obtained from the spectra themselves (grey diamonds, fitted with dotted grey curve) and the predictions for time of minimum light from SuperWASP ephemerides (the solid vertical line indicates a linear ephemeris, i.e. no period change, the dotted vertical line a quadratic one, i.e. secular period change; the two are almost coincident).

(Figs. 7 and 8). The divergence between different phase measures is greater for J160156; this is a consequence of its shorter period, more rapid predicted period-change, smaller data sets (both photometric and spectroscopic), and the longer time gap between the last archived photometry and spectroscopy. We note that in each system the deeper minimum of the light curve corresponds to the eclipse of the secondary, less massive binary component; the SuperWASP light curves were consequently refolded to locate phase 0 at the time of true primary eclipse. The resulting spectroscopic observations and derived quantities are given in Tables 1 and 2. The velocity uncertainties are those obtained with FXCOR; uncertainties in phase (using the sinusoidal fitting described above) are negligible in comparison, and were not included in subsequent modelling.

\section{Results}

The eclipsing binary modelling software PHOEBE (Prša \& Zwitter 2005), built upon the code of Wilson \& Devinney (1971),

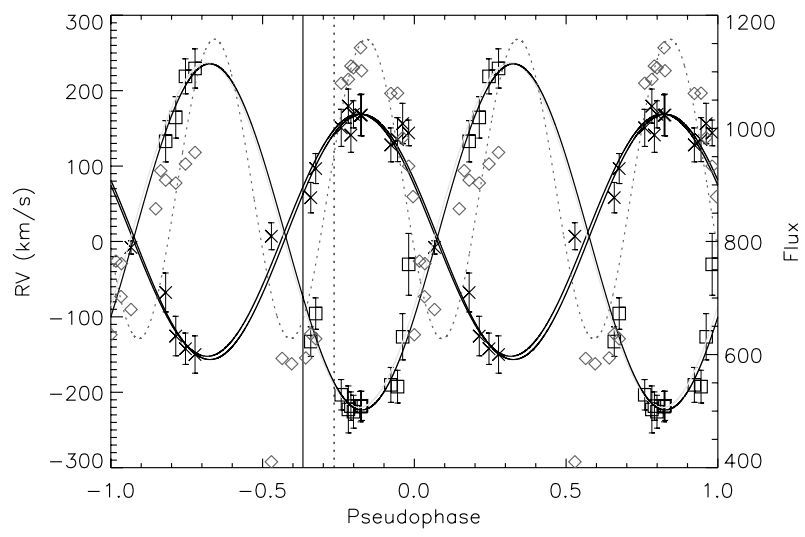

Fig. 8. Radial velocities for J160156 (crosses indicate primary component, squares secondary) with preliminary fits (solid curves) used to obtain correct phasing of observations. The primary and secondary curve fits were determined consecutively, with the second fit deriving some parameters from the first, and then refitted in the other order; this has resulted in the visibly double fit curve for the primary component. Also shown are a light curve obtained from the spectra themselves (grey diamonds, fitted with dotted grey curve) and the predictions for time of minimum light from SuperWASP ephemerides (the solid vertical line indicates a linear ephemeris, the dotted vertical line a quadratic one).

was used to model simultaneously the binned SuperWASP light curves and INT radial velocity curves of the two systems. (The full SuperWASP light curves were also modelled as a final check on the validity of the optima found using binned curves; it would have been prohibitively time-consuming to carry out the full modelling procedure using curves consisting of tens of thousands of data points.) A semi-detached or contact configuration (one or both components overfilling their Roche lobes) was assumed on the basis of the continuous light variation in the light curves, so the unconstrained mode was used to allow for both possibilities. An approximate shared temperature (which would correspond to the envelope surrounding the components of a W UMa-type system) of $4500 \pm 250 \mathrm{~K}$ was used for both EBs because a template with this effective temperature had provided the best match for phase 0 and phase 0.5 spectra during radial velocity determination; this was not varied during modelling because of the relatively low $\mathrm{S} / \mathrm{N}$ of the spectroscopic data and the limited contribution of temperature to the goodness of model fit.

The shortness of the orbital periods involved constrained us to sub-solar or approximately solar parameters for masses and radii: large stars simply would not fit into the orbits implied, and so trial values of semi-major axes were limited accordingly. No third light was included since in each light curve the deeper eclipse has roughly half the flux of the higher maximum. The details of the light curve shape also constrained the possible angles of inclination: J150822 has slightly flattened eclipse bottoms, implying $i$ close to $90^{\circ}$, while $\mathrm{J} 160156$ has more pointed eclipse bottoms, ruling out such a high angle. The shapes of the shoulders of the maxima in each case implied Kopal potentials lower than the critical potential at Lagrange point $L_{1}$, i.e. yielding binary filling factors in $(0,1]$, using Prša's definition

$F=\frac{\Omega-\Omega_{\text {crit }}^{L_{1}}}{\Omega_{\text {crit }}^{L_{2}}-\Omega_{\text {crit }}^{L_{1}}}$.

The radial-velocity curve amplitudes alone determined the semimajor axes of the orbits $a$ and hence the absolute sizes of the components, while their mass ratios $q$ were constrained by 
Table 1. Summary of spectroscopic observations and derived quantities for J150822.

\begin{tabular}{|c|c|c|c|c|c|c|}
\hline $\begin{array}{l}\text { HJD } \\
-2450000\end{array}$ & Phase & $\begin{array}{c}\text { Primary } \\
R V\left(\mathrm{~km} \mathrm{~s}^{-1}\right)\end{array}$ & $\begin{array}{c}\delta \text { Primary } \\
R V\left(\mathrm{~km} \mathrm{~s}^{-1}\right)\end{array}$ & $\begin{array}{l}\text { Secondary } \\
R V\left(\mathrm{~km} \mathrm{~s}^{-1}\right)\end{array}$ & $\begin{array}{l}\delta \text { Secondary } \\
R V\left(\mathrm{~km} \mathrm{~s}^{-1}\right)\end{array}$ & $\begin{array}{l}\text { Continuum flux at } \\
8500 \AA \text { (arb. units) }\end{array}$ \\
\hline 5997.5934 & 0.302 & $a$ & & & & 2995 \\
\hline 5997.5977 & 0.319 & -128 & 24 & 242 & 37 & 2858 \\
\hline 5997.6015 & 0.333 & -117 & 26 & 248 & 34 & 2342 \\
\hline 5997.6406 & 0.484 & & & & & 1115 \\
\hline 5997.6444 & 0.498 & & & & & 1202 \\
\hline 5997.6482 & 0.513 & & & & & 1346 \\
\hline 5997.6756 & 0.618 & 46 & 29 & -203 & 36 & 3682 \\
\hline 5997.6792 & 0.632 & 70 & 38 & -191 & 45 & 3852 \\
\hline 5997.6829 & 0.646 & 109 & 30 & -233 & 27 & 3678 \\
\hline 5997.7080 & 0.743 & 118 & 33 & -265 & 23 & 4034 \\
\hline 5997.7119 & 0.758 & 126 & 31 & -265 & 23 & 3978 \\
\hline 5997.7155 & 0.772 & 118 & 36 & -257 & 24 & 4278 \\
\hline 5998.6826 & 0.490 & & & & & 2050 \\
\hline 5998.6863 & 0.504 & & & & & 2095 \\
\hline 5998.7272 & 0.661 & 127 & 28 & -221 & 30 & 2196 \\
\hline 5998.7309 & 0.676 & 100 & 37 & -237 & 25 & 3401 \\
\hline 5998.7348 & 0.691 & 126 & 28 & -239 & 23 & 3530 \\
\hline 5998.7571 & 0.776 & 145 & 24 & -243 & 28 & 4294 \\
\hline 5998.7613 & 0.793 & 130 & 29 & -245 & 24 & 4143 \\
\hline 5998.7650 & 0.807 & 139 & 23 & -227 & 31 & 3403 \\
\hline 5999.5733 & 0.915 & 69 & 22 & -117 & 22 & 2737 \\
\hline 5999.5770 & 0.929 & & & & & 2689 \\
\hline 5999.5806 & 0.943 & & & & & 2459 \\
\hline 5999.6170 & 0.083 & -85 & 18 & 86 & 21 & 2965 \\
\hline 5999.6244 & 0.111 & -86 & 23 & 131 & 26 & 3316 \\
\hline 5999.6316 & 0.139 & -111 & 30 & 190 & 30 & 3613 \\
\hline 5999.6680 & 0.279 & -143 & 24 & 253 & 27 & 4167 \\
\hline 5999.6765 & 0.312 & -147 & 27 & 237 & 27 & 3810 \\
\hline 5999.6850 & 0.344 & -131 & 29 & 235 & 31 & 3744 \\
\hline 5999.7209 & 0.482 & & & & & 1954 \\
\hline 5999.7282 & 0.510 & 0 & 13 & & & 1754 \\
\hline 5999.7356 & 0.539 & & & & & 2014 \\
\hline 5999.7681 & 0.664 & 116 & 26 & -219 & 27 & 3669 \\
\hline 5999.7719 & 0.678 & 121 & 29 & -224 & 30 & 3737 \\
\hline 5999.7755 & 0.692 & 121 & 27 & -269 & 21 & 3582 \\
\hline 5999.7804 & 0.711 & 138 & 21 & -248 & 25 & 3802 \\
\hline
\end{tabular}

Notes. ${ }^{(a)}$ Radial velocities unusable.

both light and radial-velocity curves via the relative eclipse depths and relative amplitudes of primary and secondary components. The light curves provided most of the information needed to determine the optimum angles of inclination $i$ and Kopal potentials $\Omega_{1,2}$.

Using these guidelines and following a similar approach to that of Chew (2010), an initial best-fit solution was found manually for each system, which minimized the combined $\chi^{2}$ values for the light curve and the two radial velocity curves. To ensure that these solutions corresponded to global rather than local minima, to explore the correlations between fitting parameters, and to determine realistic uncertainties for the best-fit parameter values, a series of heuristic scans of the five-dimensional parameter space $\left(a, q, i\right.$, and $\left.\Omega_{1,2}\right)$ were carried out using the PHOEBE scripter. Initially, the entire physically plausible parameter space was scanned with widely spaced grids to ensure that no regions of low $\chi^{2}$ values had been missed. The scans were then repeated with decreasing grid spacings, focusing on regions where the difference $\Delta \chi^{2}=\chi^{2}-\chi_{\min }^{2}$ corresponded to an uncertainty lower than $3 \sigma$ (Press et al. 2007), until the position of the minimum was determined with accuracy.

The global optima found for the two systems via the scans were very close to those found manually; the combined minimum $\chi^{2}$ value for J150822 was 2.36 and for J160156 was 4.72. Since these values were much higher than 1 , indicating poor model fits (for reasons explored below), the $\Delta \chi^{2}$ value at which to set the $1 \sigma$ uncertainty boundary was set with the assistance of a separate series of manually determined best-fit solutions for simulated data sets, with data points randomly perturbed according to their original individual uncertainties. The standard deviations of the parameters, estimated by this method were comparable in size with the formal uncertainties given by the Wilson-Devinney covariance matrix.

Figures 9-16 illustrate some of the ten two-dimensional parameter cross-sections obtained from the scans. The $\Omega_{1,2}-q$ planes are particularly revealing: both systems have best-fit solutions in which both components overfill their Roche lobes, and where the two potentials are strongly correlated with each other (Figs. 12 and 16), suggesting that the components of each system share a common potential within the shared envelope of a contact binary.

Figures 17 to 28 show the best-fit PHOEBE models for the two systems. A weak Rossiter-McLaughlin effect (Rossiter 1924; McLaughlin 1924) is seen in the model for J150822 in the asymmetry of the radial velocity curves (Fig. 17); this is a consequence of the high inclination angle. The different 
M. E. Lohr et al.: Parameters of two eclipsing binaries near the short-period limit

Table 2. Summary of spectroscopic observations and derived quantities for J160156.

\begin{tabular}{|c|c|c|c|c|c|c|}
\hline $\begin{array}{l}\text { HJD } \\
-2450000\end{array}$ & Phase & $\begin{array}{c}\text { Primary } \\
R V\left(\mathrm{~km} \mathrm{~s}^{-1}\right)\end{array}$ & $\begin{array}{c}\delta \text { Primary } \\
R V\left(\mathrm{~km} \mathrm{~s}^{-1}\right)\end{array}$ & $\begin{array}{l}\text { Secondary } \\
R V\left(\mathrm{~km} \mathrm{~s}^{-1}\right)\end{array}$ & $\begin{array}{l}\delta \text { Secondary } \\
R V\left(\mathrm{~km} \mathrm{~s}^{-1}\right)\end{array}$ & $\begin{array}{l}\text { Continuum flux at } \\
8500 \AA \text { (arb. units) }\end{array}$ \\
\hline 5997.6567 & 0.923 & & & & & 635 \\
\hline 5997.6605 & 0.940 & & & & & 765 \\
\hline 5997.6643 & 0.957 & & & & & 702 \\
\hline 5997.6902 & 0.071 & & & & & 858 \\
\hline 5997.6939 & 0.087 & & & & & 925 \\
\hline 5997.6976 & 0.104 & -68 & 26 & 133 & 27 & 909 \\
\hline 5998.7083 & 0.565 & & & & & 594 \\
\hline 5998.7120 & 0.582 & 58 & 20 & -133 & 20 & 637 \\
\hline 5998.7157 & 0.598 & 97 & 20 & -96 & 21 & 628 \\
\hline 5998.7401 & 0.706 & 180 & 23 & -223 & 31 & 1087 \\
\hline 5998.7439 & 0.722 & 169 & 18 & -226 & 22 & 1107 \\
\hline 5998.7499 & 0.749 & 168 & 28 & -218 & 19 & 1102 \\
\hline 5998.7718 & 0.846 & 128 & 22 & -190 & 23 & 1062 \\
\hline 5998.7768 & 0.868 & 135 & 29 & -193 & 22 & 1062 \\
\hline 5998.7806 & 0.884 & 156 & 27 & -126 & 31 & 983 \\
\hline 5998.7851 & 0.904 & 144 & 17 & -30 & 41 & 933 \\
\hline 5999.5888 & 0.452 & 7 & 18 & & & 410 \\
\hline 5999.5968 & 0.487 & & & & & 593 \\
\hline 5999.6039 & 0.519 & & & & & 584 \\
\hline 5999.6409 & 0.682 & 151 & 25 & -203 & 20 & 1080 \\
\hline 5999.6481 & 0.714 & 141 & 23 & -218 & 19 & 1110 \\
\hline 5999.6553 & 0.746 & 167 & 27 & -219 & 20 & 1143 \\
\hline 5999.6945 & 0.919 & & & & & 879 \\
\hline 5999.7033 & 0.958 & & & & & 761 \\
\hline 5999.7105 & 0.989 & -8 & 9 & & & 680 \\
\hline 5999.7439 & 0.137 & -125 & 26 & 165 & 27 & 903 \\
\hline 5999.7512 & 0.169 & -142 & 21 & 219 & 23 & 937 \\
\hline 5999.7583 & 0.200 & -150 & 25 & 229 & 26 & 957 \\
\hline
\end{tabular}

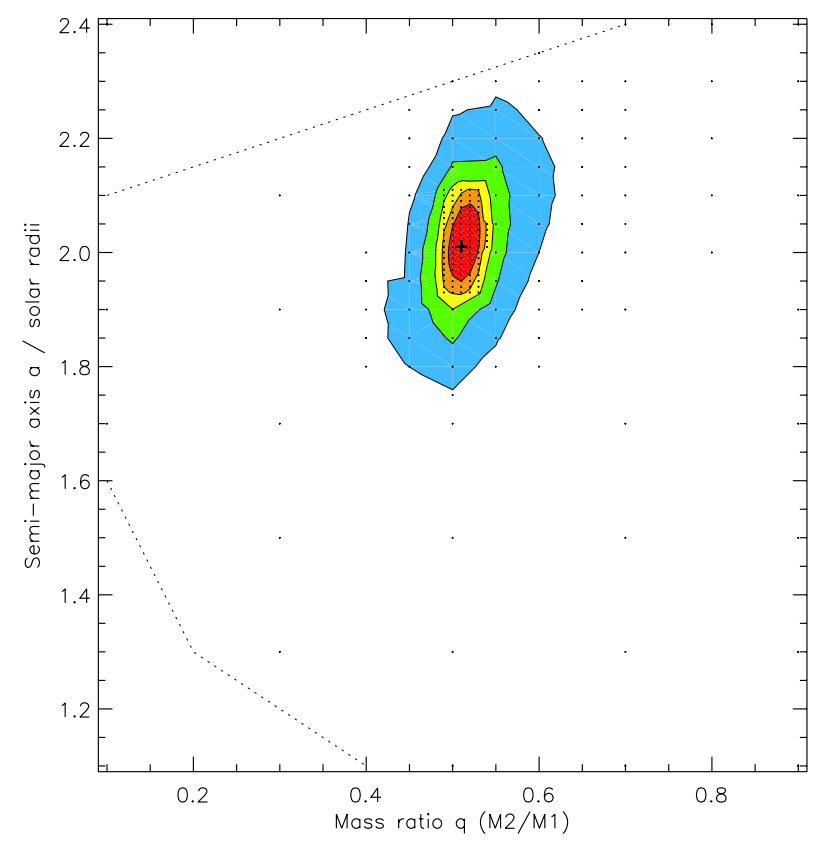

Fig. 9. $a-q$ parameter cross-section for J150822. Boxes indicate points sampled (other parameters are optimized) and the global minimum is marked with a cross. Contour lines show the 1, 2, 3, 4, and $5 \sigma$ uncertainty levels derived from the $\Delta \chi^{2}$ values of the sampled points. Points outside the plot boundaries or the dotted lines were not sampled since they corresponded to physically implausible masses for the stellar components $\left(<0.08\right.$ or $\left.>1.5 M_{\odot}\right)$.

heights of maxima visible in both light curves, but most notably in J160156, are most likely attributable to star spots, that is, the

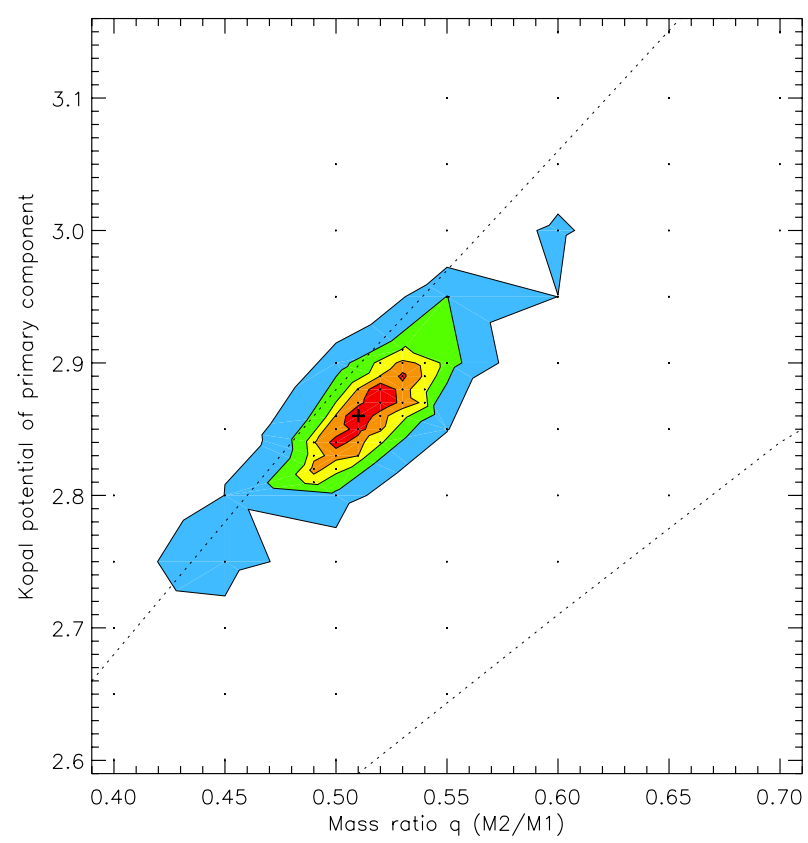

Fig. 10. $\Omega_{1}-q$ parameter cross-section for J150822. Points below the lower dotted line were not sampled since they correspond to physically implausible filling factors $(F>1)$; very high potentials, corresponding to highly unlikely detached configurations, were not sampled either. The upper dotted line indicates the location of the binary's Roche lobe; the primary component is (with high probability) just below this line, and hence probably just overfills the Roche lobe.

O'Connell effect (O'Connell 1951). However, since we lack any direct evidence for the number, size, or location of spots (e.g. 


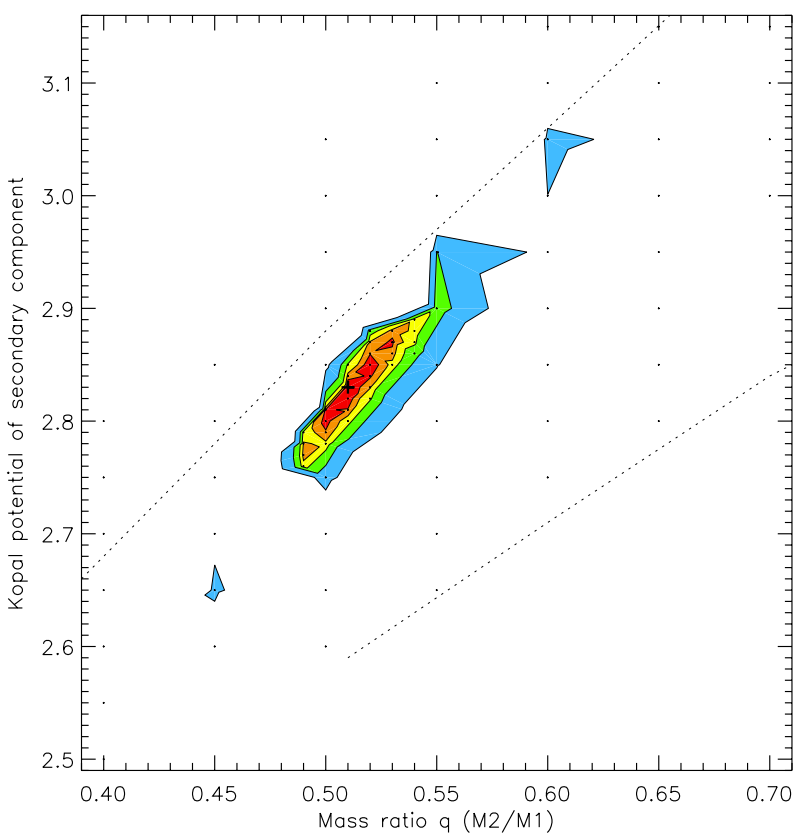

Fig. 11. $\Omega_{2}-q$ parameter cross-section for J150822 (see caption to Fig. 10 for an explanation of the dotted lines). The secondary component is, with very high probability, overfilling the Roche lobe.

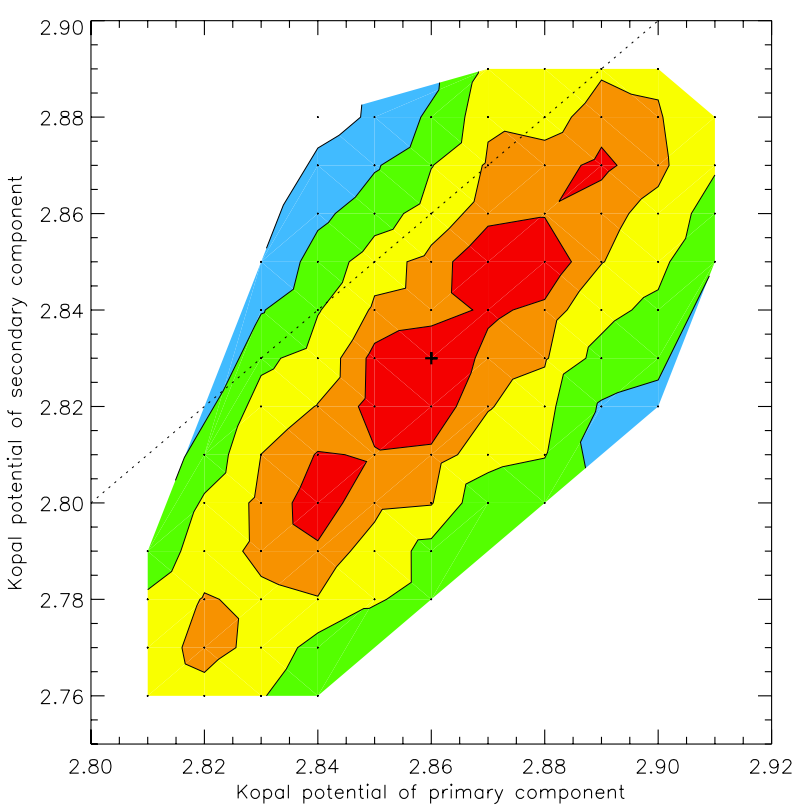

Fig. 12. $\Omega_{1}-\Omega_{2}$ parameter cross-section for J150822. The dotted line here indicates equal potentials for the two components, which would necessarily be the case in a contact system; their probability distribution almost follows this line, showing a strong correlation between $\Omega_{1}$ and $\Omega_{2}$.

via Doppler tomography), and being mindful of the additional modelling latitude provided by including spots, we sought to determine the best-fit model for the light curves without including any spots, and our final stellar parameters result from this model. Figures 20 and 26 indicate the improvement of fit resulting from adding a single cool spot to the primary component in each system without altering any other input parameters. Figures 22 and 28 show the appearance of the modelled spotted systems, which reproduce both the different heights of maxima and the different depths of minima better than the unspotted

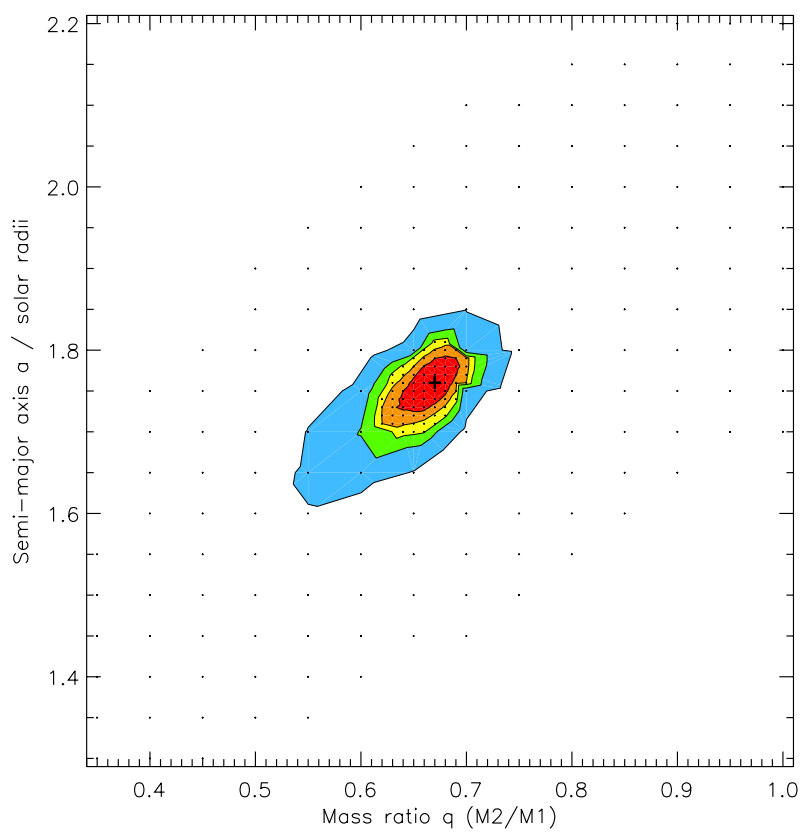

Fig. 13. $a-q$ parameter cross-section for J160156. A fairly strong correlation between semi-major axis and mass ratio is apparent.

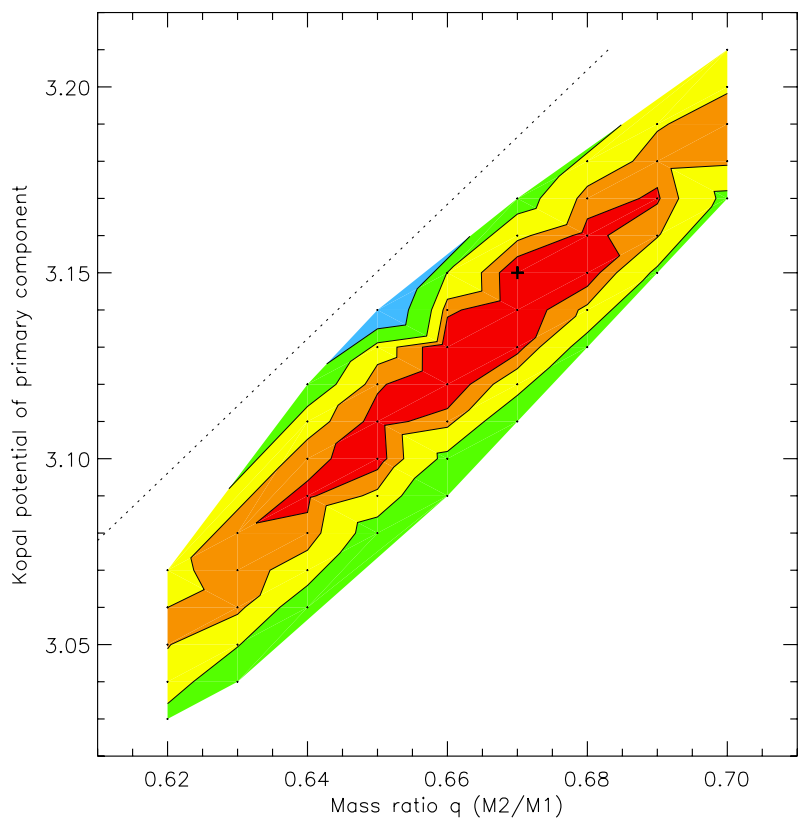

Fig. 14. $\Omega_{1}-q$ parameter cross-section for J160156. The dotted line indicates the location of the binary's Roche lobe; the primary component is with high probability below this line, and hence very likely overfills the Roche lobe.

models can. Addition of more spots might doubtless produce a perfect match of models to light curves, but at the expense of the plausibility of the modelling.

The residuals for the light curve fits reflect these assumed spots in their large-scale sinusoidal deviations (Figs. 19 and 25). However, there are additional clear sinusoidal variations at a smaller scale in the residuals for J150822 (Fig. 21): an oscillation with an amplitude of around \pm 0.1 flux units and a frequency of six cycles per orbit. Presumably these correspond to pulsation of the primary (since they are obscured during primary eclipse), and are locked to the binary orbital period (since they are clearly visible in the folded light curve). 
M. E. Lohr et al.: Parameters of two eclipsing binaries near the short-period limit

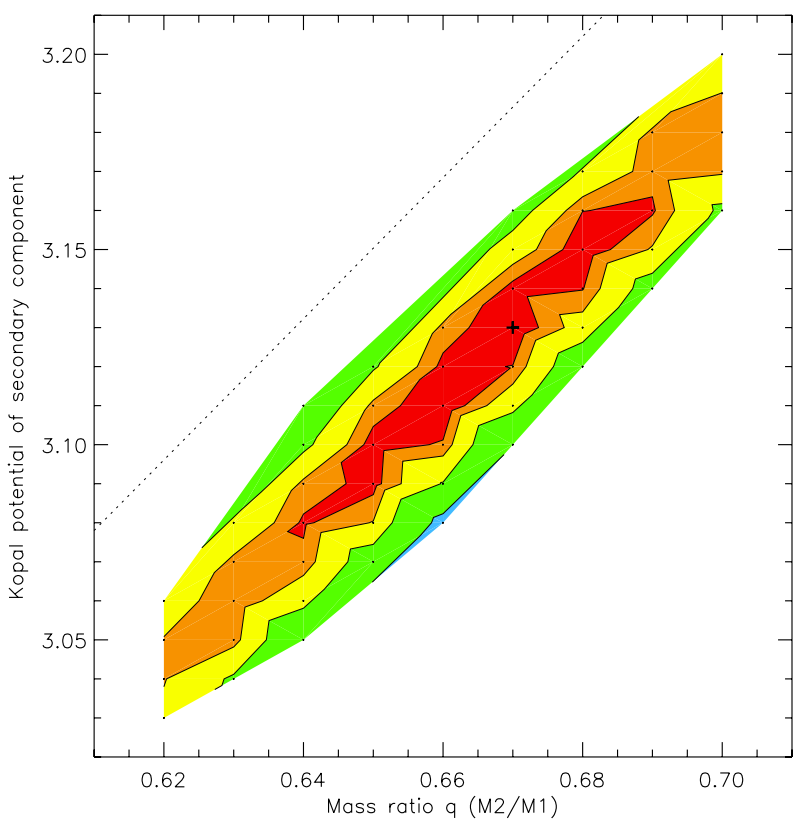

Fig. 15. $\Omega_{2}-q$ parameter cross-section for J160156. The secondary component is also very likely overfilling the Roche lobe.

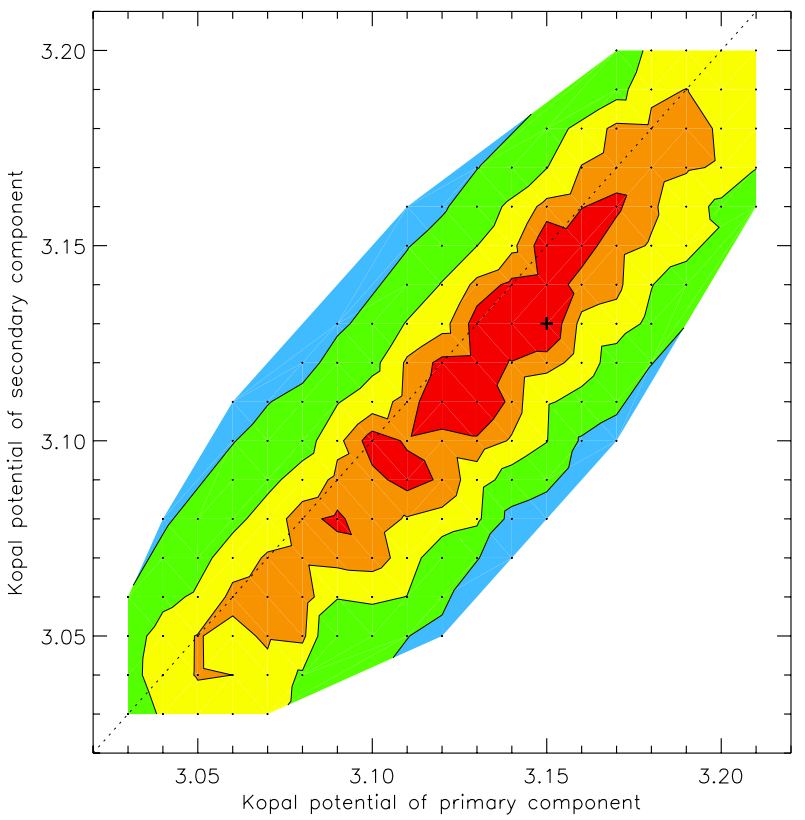

Fig. 16. $\Omega_{1}-\Omega_{2}$ parameter cross-section for J160156. The region of highest probability closely follows the dotted line, suggesting nearidentical potentials for the two components.

The final best-fit parameters for both systems are given in Table 3. We would emphasise that these parameters are not dependent on the inclusion of spots in the models. The uncertainties on $a, q, i$ and $\Omega_{1,2}$ were obtained from the $1 \sigma$ contours in the relevant parameter cross-sections. The uncertainties on the output parameters (masses and radii) are the maximum/minimum values obtainable using parameter combinations falling within these $1 \sigma$ contours.

\section{Discussion}

These results confirm J150822 and J160156, initially identified as candidate EBs on the basis of light curve shapes alone, as

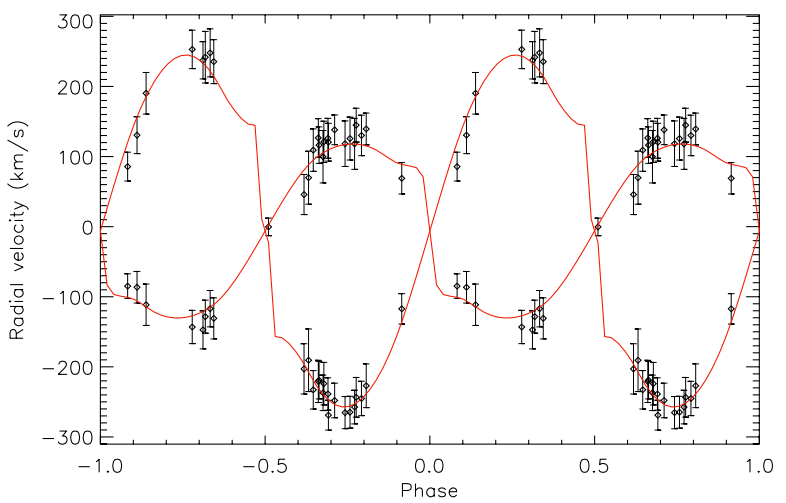

Fig. 17. Radial velocity curves for J150822 with the best-fit model overplotted.

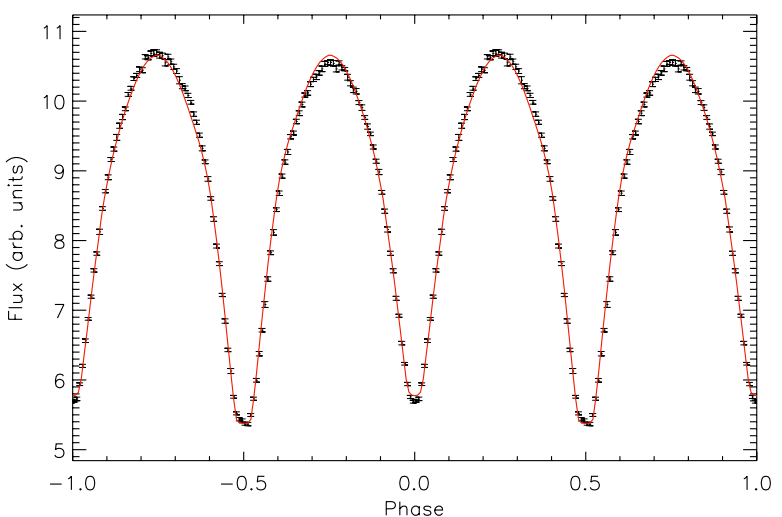

Fig. 18. SuperWASP binned light curve for J150822 with the best-fit un-spotted model overplotted.

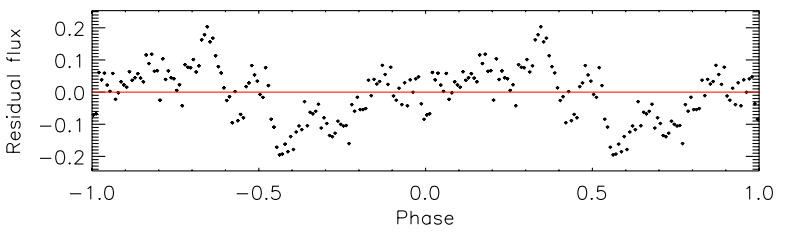

Fig. 19. Light-curve residuals for the J150822 best-fit model.

Table 3. Modelled system and stellar component parameters for $\mathrm{J} 150822$ and $\mathrm{J} 160156$.

\begin{tabular}{lccc}
\hline \hline & & $\mathrm{J} 150822$ & $\mathrm{~J} 160156$ \\
\hline Semi-major axis $\left(R_{\odot}\right)$ & $a$ & $2.01_{-0.06}^{+0.07}$ & $1.76 \pm 0.03$ \\
Mass ratio & $M_{2} / M_{1}$ & $0.51_{-0.01}^{+0.02}$ & $0.67_{-0.03}^{+0.02}$ \\
COM velocity $\left(\mathrm{km} \mathrm{s}^{-1}\right)$ & $V_{0}$ & $-6.2_{-2.5}^{+2.8}$ & $4.7_{-1.2}^{+1.8}$ \\
Angle of incl. $\left(^{\circ}\right)$ & $i$ & $90_{-3}^{+0}$ & $79.5 \pm 0.25$ \\
Kopal potentials & $\Omega_{1}$ & $2.86_{-0.02}^{+0.03}$ & $3.15_{-0.06}^{+0.02}$ \\
& $\Omega_{2}$ & $2.83 \pm 0.04$ & $3.13_{-0.05}^{+0.03}$ \\
Filling factor & $\mathcal{F}$ & $0.12_{-0.04}^{+0.06}$ & $0.10_{-0.00}^{+0.06}$ \\
Masses $\left(M_{\odot}\right)$ & $M_{1}$ & $1.07_{-0.09}^{+0.12}$ & $0.86 \pm 0.04$ \\
& $M_{2}$ & $0.55_{-0.05}^{+0.06}$ & $0.57 \pm 0.04$ \\
Radii $\left(R_{\odot}\right)$ & $R_{1}$ & $0.90_{-0.03}^{+0.04}$ & $0.75 \pm 0.01$ \\
& $R_{2}$ & $0.68 \pm 0.03$ & $0.63 \pm 0.02$ \\
\hline
\end{tabular}

double-lined spectroscopic and eclipsing binaries. From modelling, both systems appear to be composed of late G-early $\mathrm{M}$ class dwarfs. J150822 is slightly more massive, with an approximately solar-mass primary and late $\mathrm{K}$ secondary; its masses have been determined with a precision of $\sim 10 \%$ and its radii 


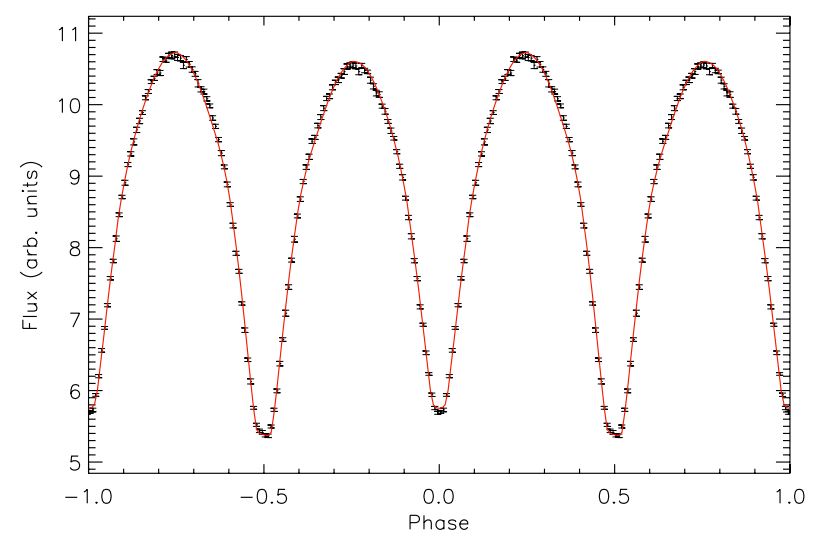

Fig. 20. Best-fit model for J150822 with example spot included.

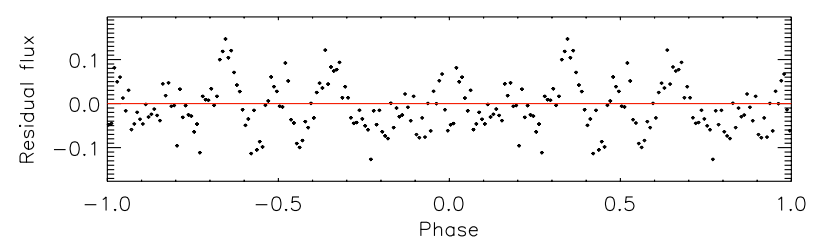

Fig. 21. Light-curve residuals for the J150822 example model with a single cool spot.

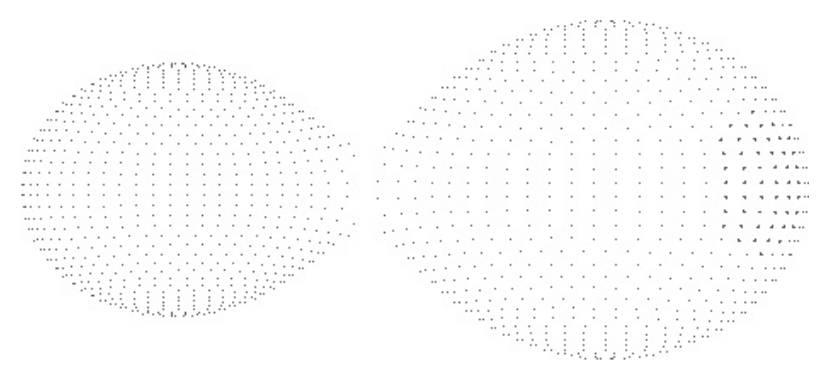

Fig. 22. Image of J150822 PHOEBE best-fit model at phase 0.75 , indicating location and size of example cool spot on primary.

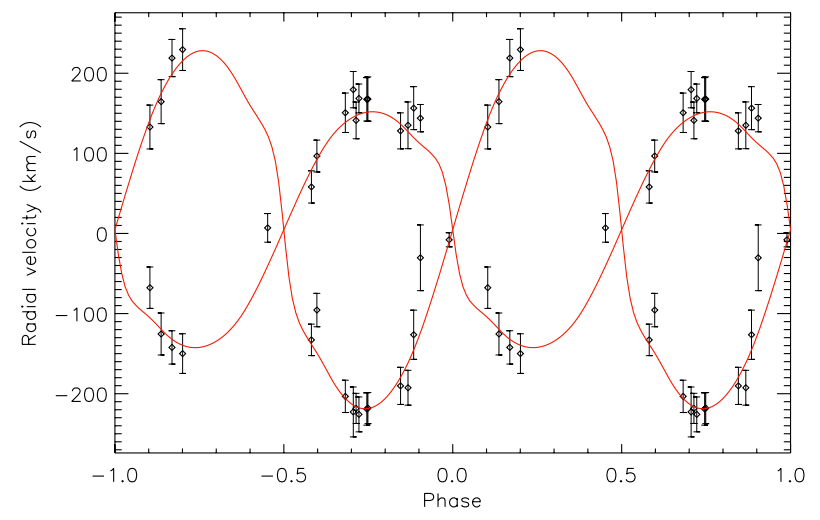

Fig. 23. Radial velocity curves for J160156 with the best-fit model overplotted.

within $\sim 4 \%$. The components of J160156 are of more similar mass: a late-G or early-K primary and a late-K or early-M secondary; its masses have been found with a precision of $\sim 5 \%$ and its radii within $\sim 2 \%$. Both appear to be $\mathrm{W}$-type systems, in the sense of Binnendijk (1970), in that the less massive component is eclipsed during the deeper minimum.

The contact configuration and likely mass exchange associated with the apparent period changes make it difficult to

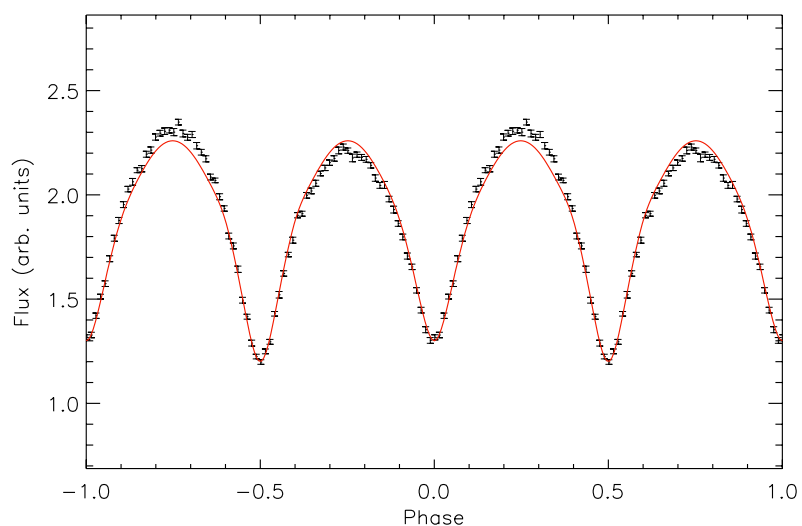

Fig. 24. SuperWASP binned light curve for J160156 with best-fit unspotted model overplotted.

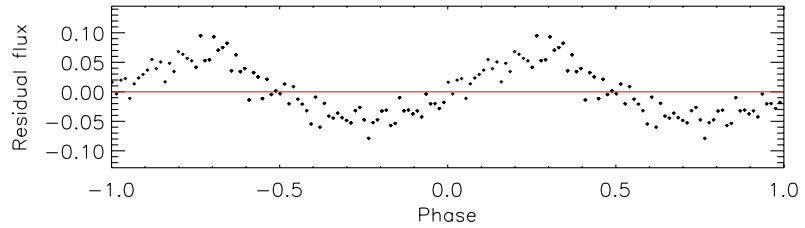

Fig. 25. Light curve residuals for J160156 best-fit model.

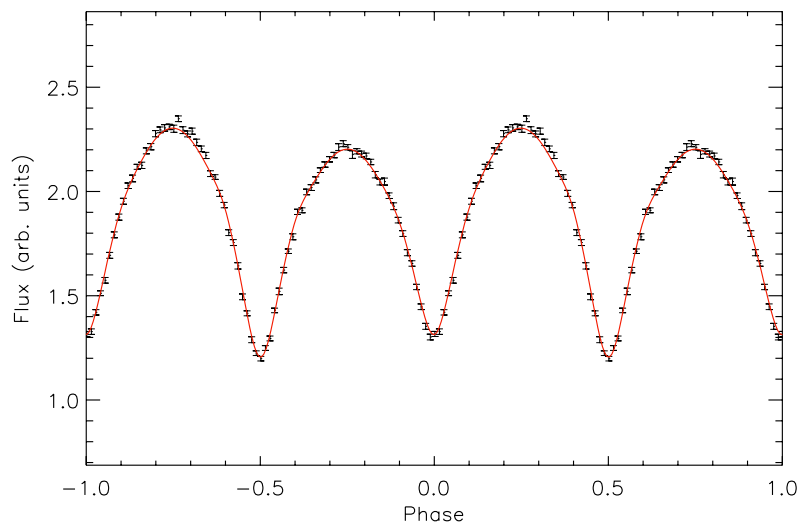

Fig. 26. Best-fit model for J160156 with example spot included.

compare directly the mass-radius relationships of these systems with those collected and discussed for example in Torres et al. (2010, Fig. 2) and Torres (2013, Fig. 4) for detached binaries containing low-mass components. Figure 29 therefore compares our results with those of Torres et al. and of a selection of short-period contact systems collected by Stepień \& Gazeas (2012). We note that like several other contact systems, the binaries studied here are somewhat discrepant with the Dartmouth model isochrones for solar metallicity (Dotter et al. 2008) ${ }^{1}$ models. Specifically, the primaries have smaller radii than their masses might suggest, while the secondaries have larger radii than expected. Possibly the primaries' less dense outer layers have been partially stripped and transferred to the secondaries, leaving denser "cores". Higher resolution spectroscopy and/or Doppler tomography would be required for confirmation.

\section{Conclusions}

J150822 and J160156 are established to be spectroscopic double-lined and eclipsing binary systems in contact

1 http://stellar.dartmouth.edu/ 
M. E. Lohr et al.: Parameters of two eclipsing binaries near the short-period limit

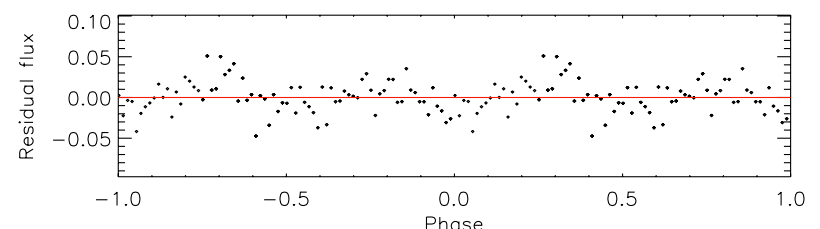

Fig. 27. Light curve residuals for J160156 example model with single cool spot.
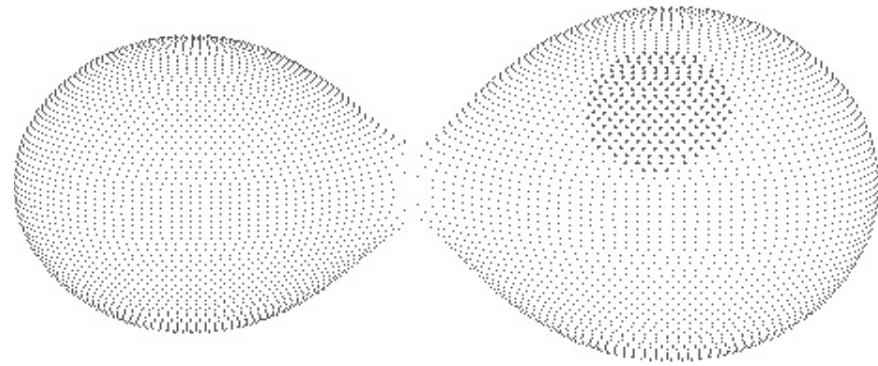

Fig. 28. Image of J160156 PHOEBE best-fit model at phase 0.75, indicating location and size of example cool spot on primary.

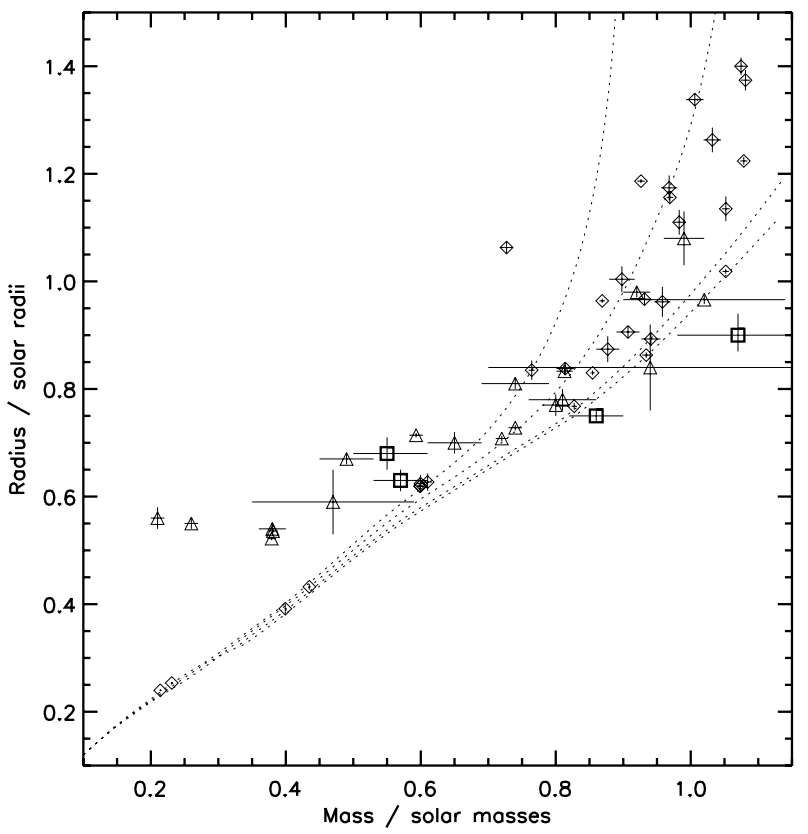

Fig. 29. Masses vs. radii of J150822 and J160156 components (squares) compared with 32 components of low-mass detached binaries (diamonds) from Torres et al. (2010) and 20 components of short-period contact binaries (triangles) from Stepień \& Gazeas (2012). Also plotted are theoretical isochrones derived from Dartmouth models (Dotter et al. 2008) for solar metallicity, with ages of $0.25,1,5$, and $10 \mathrm{Gyr}$, respectively (dotted lines, ascending).

configuration, composed of low-mass dwarfs. J150822 was modelled as consisting of components of 1.07 and $0.55 M_{\odot}$ (mass ratio 0.51 ), and $\mathrm{J} 160156$ as having components of 0.86 and $0.57 M_{\odot}$ (mass ratio 0.67 ). The primary of $\mathrm{J} 150822$ appears to be pulsating with a period of $1 / 6$ of the orbital period. Both systems are plausibly undergoing mass transfer; this may be related to the primaries' radii being smaller and the secondaries' radii being larger than would be typical for single stars with these masses.

The parameters obtained here contribute to our understanding of low-mass stars and contact binary systems, since relatively few binaries are known with such short orbital periods. We hope to follow-up more of the candidate short-period EBs listed in Lohr et al. (2013) with multi-colour photometry and spectroscopy, with a view to confirming their binary nature. Many of them are expected to be good prospects for full solution, and capable of significantly extending our knowledge of this aspect of the field.

Acknowledgements. The WASP project is funded and operated by Queen's University Belfast, the Universities of Keele, St. Andrews, and Leicester, the Open University, the Isaac Newton Group, the Instituto de Astrofísica de Canarias, the South African Astronomical Observatory, and by STFC. The Isaac Newton Telescope is operated on the island of La Palma by the Isaac Newton Group in the Spanish Observatorio del Roque de los Muchachos of the Instituto de Astrofísica de Canarias. This work was supported by the Science and Technology Funding Council and the Open University. We would like to thank the referee for constructive comments and recommendations that have improved this paper.

\section{References}

Binnendijk, L. 1970, Vistas in Astronomy, 12, 217

Chew, Y. G. M. 2010, Ph.D. Thesis; Vanderbilt University, Nashville, Tennessee, USA

Dotter, A., Chaboyer, B., Jevremović, D., et al. 2008, ApJS, 178, 89

Jiang, D., Han, Z., Ge, H., Yang, L., \& Li, L. 2012, MNRAS, 421, 2769

Lohr, M. E., Norton, A. J., Kolb, U. C., et al. 2012, A\&A, 542, A124

Lohr, M. E., Norton, A. J., Kolb, U. C., et al. 2013, A\&A, 549, A86

Mazeh, T., Tamuz, O., Zucker, S., et al. 2006, in Proc. Colloq. Tenth Anniversary of 51 Peg-b: Status of and prospects for hot Jupiter studies, eds. L. Arnold, F. Bouchy, \& C. Moutou (Paris: Frontier Group), 165, available at http:// obs-hp.fr/www/pubs/Coll51Peg/proceedings.html

McLaughlin, D. B. 1924, ApJ, 60, 22

Norton, A. J., Payne, S. G., Evans, T., et al. 2011, A\&A, 528, A90

O'Connell, D. J. K. 1951, Publications of the Riverview College Observatory, 2, 85

Paczyński, B., Szczygieł, D. M., Pilecki, B., \& Pojmański, G. 2006, MNRAS, 368,1311

Pollacco, D. L., Skillen, I., Cameron, A. C., et al. 2006, PASP, 118, 1407

Press, W. H., Teukolsky, S. A., Vetterling, W. T., \& Flannery, B. P. 2007, Numerical Recipes: The Art of Scientific Computing, 3rd edn. (New York: Cambridge University Press)

Prša, A., \& Zwitter, T. 2005, ApJ, 628, 426

Rossiter, R. A. 1924, ApJ, 60, 15

Rucinski, S. M. 1992, AJ, 103, 960

Rucinski, S. M. 2007, MNRAS, 382, 393

Stepień, K. 2006, Acta Astron., 56, 347

Stepień, K., \& Gazeas, K. 2012, Acta Astron., 62, 153

Szymański, M., Kubiak, M., \& Udalski, A. 2001, Acta Astron., 51, 259

Tamuz, O., Mazeh, T., \& Zucker, S. 2005, MNRAS, 356, 1466

Torres, G. 2013, Astron. Nachr., 334, 4

Torres, G., Andersen, J., \& Giménez, A. 2010, A\&AR, 18, 67

Wilson, R. E., \& Devinney, E. J. 1971, ApJ, 166, 605 